\title{
A PDE APPROACH OF INFLAMMATORY PHASE DYNAMICS IN DIABETIC WOUNDS
}

\author{
N. Cónsul ${ }^{*}$, S. M. Oliva ${ }^{\dagger}$, And M. Pellicer ${ }^{\ddagger}$
}

\begin{abstract}
The objective of the present paper is the modeling and analysis of the dynamics of macrophages and certain growth factors in the inflammatory phase, the first one of the wound healing process. It is the phase where there exists a major difference between diabetic and non-diabetic wound healing, an effect that we will consider in this paper.
\end{abstract}

2010 Mathematics Subject Classification: 35Q92, 35A02, 65P30.

Key words: Wound healing modelling, diabetes influence, analytical well-posedness, bifurcation diagrams, numerical analysis of equilibria.

\section{Introduction}

Wound healing is an extremely complicated process and still not fully understood. Even more when a disease, such for instance diabetes, is present. The present work aims at contributing to this analysis by focusing on the study of one of the parts of the whole process, the inflammatory phase, in the case of possibly diabetic patients. We will propose and analyze a partial differential equation as a model for the interaction of some of the crucial elements involved in this phase of the wound healing. This model will generalize a previous existing ODE model.

Let us begin with a description of the part of the wound healing process that we are interested in: the inflammatory phase. When a tissue injury occurs, even a superficial one, a cascade of events begins. This process is usually divided in three stages, which overlap in time: the inflammatory phase, the proliferative (or tissue formation) phase and the tissue remodeling phase.

\footnotetext{
*Supported by grants MTM2011-27739-C04-01 (Spain) and 2009SGR345 (Catalonia). ${ }^{\dagger}$ Supported by FAPESP 2008/55516-3 and CAPES/DGU 238/11 (Brazil).

¥Supported by grants MTM2011-27739-C04-03 (Spain) and 2009SGR345 (Catalonia).
} 
As we have said, we are interested in the first of them, the inflammatory one, where essentially the whole wound healing process becomes activated. It is in this phase were the effect of the diabetes is more present and can even become critical. For a more technical and complete description of this part, see [15] and [13] and the references therein. Just to have a brief insight to what occurs and roughly speaking, what happens after an injury occurs is the following. First, the platelets are immediately activated: they must plug the wound in order not to loose more blood, but they are also responsible for recruiting some growth factors to the wound site. These factors act as chemical signals for different types of cells. The one that mainly interests us is the so-called transforming growth factor- $\beta$ (TGF- $\beta$ ). It recruits monocytes, a type of cells that form part of the immune system, that once at wound site are transformed into macrophages. Their function is to phagocytose bacteria, damaged tissue and debris to clean the wound site. Also, they produce different types of growth factors in order to continue the process and to be able to begin the other phases of the healing.

More concretely, once at the wound site the monocytes become differentiated into inflammatory and repair macrophages. The first ones fabricate TGF- $\beta$, that again recruits more monocytes to the wound site and, hence, the cycle continues. The other ones fabricate other agents in order to be able to pass to the next phase of the healing process. We want to focus on the dynamics of this inflammatory phase once the very first activation of the process has occurred and, therefore, we are in this kind of cycle.

Observe that in this inflammatory phase no tissue replacing occurs, but only the activation of the process that we have just briefly described. In order to begin the next phase of the healing process, the balance between both types of macrophages becomes crucial.

As we said before, this normal process can be disrupted in some way by several causes. One of them is diabetes. It has been observed that for those patients, the inflammatory phase takes longer and can even become chronic. This happens because the diabetes changes the proportion in which monocytes are differentiated into inflammatory or repair macrophages. This fact may make it difficult to achieve the correct balance between both types of macrophages in order to pass to the next phase and continue the normal healing process.

There exist several works related to the modeling of some part of the wound healing process. However, almost all the mathematical models for wound healing concern the repair phase (as in J. A. Sherratt and J. D. Murray [12], R. T. Tranquillo and J. D. Murray [14], L. Olsen 
et al. [9], and McDougall et al. [8]). This paper builds on the work of H. V. Waugh and J. A. Sherratt (see [15] and [16]) which concerns the earlier inflammation phase of healing, for which the diabetic profile of the patient is also taken into account. In [15], the authors present an ODE model to describe the interaction between inflammatory and repair macrophages and TGF- $\beta$, three crucial agents in the inflammatory phase in the case of a diabetic patient. We denote by $u(t)$ the inflammatory macrophage density, by $v(t)$ the repair macrophage density and by $T(t)$ the TGF- $\beta$ concentration. Then, the model in $[\mathbf{1 5}]$ is the following one:

$$
\left\{\begin{array}{l}
u_{t}=\alpha K(T)+\beta u(1-\gamma(u+v))-\delta_{1} u \\
v_{t}=(1-\alpha) K(T)+\beta v(1-\gamma(u+v))-\delta_{1} v \\
T_{t}=\eta u-\delta_{2} T
\end{array}\right.
$$

where $\alpha \in[0,1]$ represents the diabetic parameter, and the production, natural loss and competition effects have been considered. A complete explanation of the meaning of all the terms and the parameters appearing in this model will be done in Section 2 below (and, obviously, can also be found in [15]). However, let us comment some results related with the diabetic parameter $\alpha$ that can be found in [15]. In the previous ODE model, the simulation of a non-diabetic healing is done when $\alpha=0.5$ (observe that in this situation we have a balance between both types of macrophages in (1.1)). In this case, this model has a unique equilibrium, which is stable and is below a certain threshold value and, hence, allows to enter the next phase of the healing process. In this sense, we can say that all the solutions tend to this healed state.

On the other hand, when $\alpha$ is increased this balance is disrupted as the income of inflammatory macrophages is increased. According to [15], $\alpha=0.8$ will simulate the diabetic healing. In this case, [15] shows the presence of three equilibria (two of them stable and one unstable). Concerning the two stable ones, we can see that one is still below a certain threshold (hence, this is still a healed state) but the second stable equilibrium shows a persistence of the inflammatory macrophages and, therefore, the next healing phase cannot start. In this sense, it can be said that in the diabetic case we can have both types of limit behaviour, depending on the initial state of the wound: we can achieve either a healed or non-healed equilibrium.

Here we want simply to emphasize the fact that, although only three elements of the process are considered, this approach suffices to get a first insight to this complicated process in wound healing. Also, it explains 
some important dynamics as, for instance, the persistence of inflammatory macrophages in diabetic patients.

In the later work [16], the authors increase the number of elements taken into account, but the philosophy is still the same.

However, in both papers the authors use an ODE approach and, hence, the space variable does not play any role. That is why, for instance, we cannot consider some important aspects of the wound healing such as the wound shape or size, possible differences in the quality or rate of healing in different parts of the wound or, of course, the different roles that the boundary and the interior of the wound may play. Consequently, a model aiming at reproducing these non-homogeneous aspects of wound healing must be a PDE. This is the type of model we are going to obtain, that pretends to be an extension of the ODE model of [15] for a non-homogeneous-in-space wound. As we will see, our work incorporates the main aspects of the work in [15] and generalizes its results. This will be done in Section 2 below. To our knowledge, this is the first time that a PDE has been proposed as a simplified model to give some insight of the inflammatory phase of the wound healing process in the presence of diabetes. This is, to our opinion, the main novelty of this work. It has to be said that there are several works on PDE models for inflammatory processes (see for instance $[\mathbf{2}]$ or $[\mathbf{3}]$ ) or for wound healing processes (as the works cited some paragraphs above), but not to the very specific problem we are dealing with.

Once we have established the PDE model, we will show in Section 3 that it is a well posed problem both from a mathematical and biological point of view. More precisely, we will prove that there exists a bounded invariant set where all the solutions are global and positive.

In Section 4 we will numerically establish some bifurcation diagrams fixing most of the parameters and moving one of them. We will get a hysteresis phenomenon that agrees with the previous mentioned results of [15] related with the parameter $\alpha$ but also extends them, giving additional information to the healing of the wound at the inflammatory phase. Hence, the use of the PDE model will be justified. These new results will be in concordance with what could be expected from a biological point of view.

Finally, in Section 5 we summarize the main conclusions, based on the results given through all the work. 


\section{The PDE model}

Let $\Omega$ denote the wound domain and be a bounded subset of $\mathbb{R}^{n}$, $n=1,2$, with smooth boundary. We now denote by $u(x, t)$ the inflammatory macrophage density, by $v(x, t)$ the repair macrophage density and by $T(x, t)$ the TGF- $\beta$ concentration, where $x \in \Omega, t \in(0, \infty)$. According to the process explained above and inspired in [15], the model we propose is the following parabolic weakly coupled system with nonlinear reaction terms in the interior and at the boundary:

$$
\begin{cases}u_{t}=D_{1} \Delta u+\xi \alpha(x) K(T)+\beta u(1-\gamma(u+v))-\delta_{1} u & \text { in } \Omega \times \mathbb{R} \\ v_{t}=D_{1} \Delta v+\xi(1-\alpha(x)) K(T)+\beta v(1-\gamma(u+v))-\delta_{1} v & \text { in } \Omega \times \mathbb{R} \\ T_{t}=D_{2} \Delta T+\eta u-\delta_{2} T & \text { in } \Omega \times \mathbb{R} \\ D_{1} \frac{\partial u}{\partial n}=(1-\xi) H \alpha(x) K(T) & \text { on } \partial \Omega \times \mathbb{R} \\ D_{1} \frac{\partial v}{\partial n}=(1-\xi) H(1-\alpha(x)) K(T) & \text { on } \partial \Omega \times \mathbb{R} \\ D_{2} \frac{\partial T}{\partial n}=-\sqrt{D_{2} \delta_{2}} T & \text { on } \partial \Omega \times \mathbb{R} .\end{cases}
$$

This system is a model for the main dynamics in the inflammatory phase some time after the tissue has been injured and the very first activation of the process has occurred, not immediately (according to [15] this means one day after the injury has happened). Except for the Laplacian terms and the boundary conditions, the other terms do appear in the model of [15] (some in a slightly different form). We are going to explain them here for a better comprehension of the proposed model.

Inflammatory macrophages. The first term is $D_{1} \Delta u$, which represents the diffusion of macrophages within the wound site with a diffusion coefficient $D_{1}>0$.

The second one, $\xi \alpha(x) K(T)$, can be seen as an influx of inflammatory macrophages term. As we said, the macrophages are produced in response to the TGF- $\beta$ and according to a certain function $K$, as in [15]. We want to remark that this function $K$ used in [15] has been calculated using empirical data. A qualitative form of $K(T)$ representing monocyte migration can be seen in Figure 2.1. Note that when $T=0$ the macrophage migration is small but positive because there is a low background level of macrophage migration. As $T$ increases, this migration initially increases, reaches a maximum and then decreases again. 

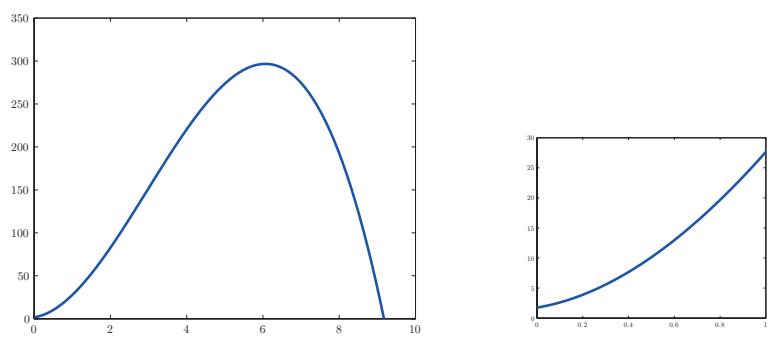

Figure 2.1. The function $K(T)$ : on the left, in the whole domain; on the right, with a zoom where the small amount of migration when $T=0$ can be observed.

The coefficient $\alpha(x) \in[0,1]$ accompanying $K(T)$ is a generalization of the diabetic parameter of the one used in [15], that indicates the proportion of monocytes becoming inflammatory macrophages. This proportion changes in the presence of diabetes, with $\alpha_{\text {diabetic }}>\alpha_{\text {healthy }}$. In $[\mathbf{1 5}], \alpha_{\text {diabetic }}=0.8$ and $\alpha_{\text {healthy }}=0.5$ are the values used for the simulations. The novelty now is that we take into account the possible dependence on $x$ of this parameter.

This second term appears with a parameter $\xi \in[0,1]$ in order to take into account the possible two places of macrophages production: at the wound site, but also outside the wound and going into the wound site through the boundary. Hence, the parameter $\xi$ represents, in some sense, the part of the reaction that takes place inside the wound or at the wound edge: if $\xi=1$ we are considering only interior production; if $\xi=0$, the production happens only at the boundary.

The next term, $\beta u(1-\gamma(u+v))$, is a growth term that takes into account the mitosis of macrophages together with the regulation of their population due to the competition between both types of macrophages. The last term $\delta_{1} u$ represents the natural loss of the inflammatory macrophages.

As a first approximation, since the boundary conditions represent the influx of macrophages into the wound through its boundary, we will consider the boundary condition as a weighted reaction $(1-\xi) H \alpha(x) K(T)$, where $H$ only takes into account the dimensional adjustment.

Repair macrophages. The equation and boundary condition for the repair macrophages includes the same type of terms as the ones for the 
inflammatory macrophages. The only difference is that the proportion of monocytes becoming repair cells is now $1-\alpha(x)$.

TGF- $\boldsymbol{\beta}$. The equation is simpler than the previous ones. This factor diffuses with a coefficient $D_{2} \gg D_{1}$ (the macrophages one), is produced by $u$, and has a natural loss, given by $\delta_{2}$ (which is of the same order as $\left.D_{2}\right)$.

As for the boundary condition of $T$, first notice that the wound edge is not really a physical boundary for $T$. Actually, the dynamics of $T$ occur in the whole space:

$$
\frac{\partial T}{\partial t}=D_{2} \Delta T-\delta_{2} T+\mathcal{X}_{\Omega}(x) \eta u \quad \text { in } \mathbb{R}^{n}
$$

with $\mathcal{X}_{\Omega}(x)$ being the characteristic function of the wound $\Omega$. As the dynamics of $T$ are fast (because $D_{2}, \delta_{2} \gg 1$ ), $T$ can be approximated outside the wound by its equilibrium solution, that is

$$
0=D_{2} \Delta T-\delta_{2} T \quad \text { in } \mathbb{R}^{n} \backslash \Omega .
$$

This is a standard approximation in biology applications (see [11] for instance). To justify the boundary condition, consider as an illustration the one-dimensional case, with a wound domain $\Omega=[0, L], L>0$. In this situation, the equilibrium solution outside the wound satisfies

$$
0=D_{2} T_{x x}-\delta_{2} T \quad \text { in }(-\infty, 0) \cup(L, \infty) .
$$

Multiplying by $T_{x}$ both sides of the equality, integrating along $(-\infty, 0)$ and assuming that

$$
\lim _{x \rightarrow \pm \infty} T(x)=0 \quad \text { and } \quad \lim _{x \rightarrow \pm \infty} T_{x}(x)=0
$$

we obtain

$$
\begin{aligned}
0 & =\int_{-\infty}^{0} D_{2} T_{x x} T_{x} d x-\int_{-\infty}^{0} \delta_{2} T T_{x} d x \\
& =\int_{-\infty}^{0} \frac{D_{2}}{2}\left(T_{x}^{2}\right)_{x} d x-\int_{-\infty}^{0} \frac{\delta_{2}}{2}\left(T^{2}\right)_{x} d x \\
& =\frac{D_{2}}{2} T_{x}^{2}(0)-\frac{\delta_{2}}{2} T^{2}(0) .
\end{aligned}
$$

Hence,

$$
T_{x}(0)= \pm \sqrt{\frac{\delta_{2}}{D_{2}}} T(0) .
$$


The same argument applies at $x=L$. Observe now that the outer normal derivative of $T$ has to be negative if (2.2) has to be fulfilled. Hence,

$$
\left.\frac{\partial T}{\partial n}\right|_{x=0, x=L}=-\left.\sqrt{\frac{\delta_{2}}{D_{2}}} T\right|_{x=0, x=L} .
$$

As we want $T$ and $T_{x}$ to be continuous in the whole real line (also at the wound edge $x=0, x=L$ ) it is reasonable to impose this condition as the boundary condition for $T$. Similarly for the two-dimensional case.

The parameters that we do not explicitly mention have the natural biological meaning and will be taken as strictly positive. For a complete description of all them and their values, see (A.1) in the appendix.

The proposed model has to deal with too many parameters. In order to avoid this and to keep only the important ones, we propose the following non-dimensionalization for Equation (2.1). We denote by ${ }^{*}$ the new variables

$$
u^{*}=\gamma u, \quad v^{*}=\gamma v, \quad T^{*}=\frac{\beta \gamma}{\eta} T, \quad t^{*}=\beta t, \quad x^{*}=\sqrt{\frac{\beta}{D_{1}}} x .
$$

Also, we denote

$$
K^{*}=\frac{\gamma}{\beta} K\left(\frac{\eta}{\beta \gamma} T^{*}\right)
$$

and the new nondimensional parameters

$$
\delta_{1}^{*}=\frac{1}{\beta} \delta_{1}, \quad \delta_{2}^{*}=\frac{1}{\beta} \delta_{2}, \quad D^{*}=\frac{D_{2}}{D_{1}}, \quad H^{*}=H \sqrt{\frac{\beta}{D_{1}}} .
$$

Observe that the new parameters and variables are non-dimensional. Using the non-dimensional changes (2.3), (2.4), and (2.5), Equation (2.1) in the new variables and parameters (that now we denote without ${ }^{*}$ for simplicity) can be written as:

$$
\begin{cases}u_{t}=\Delta u+\xi \alpha(x) K(T)+u(1-(u+v))-\delta_{1} u & \\ v_{t}=\Delta v+\xi(1-\alpha(x)) K(T)+v(1-(u+v))-\delta_{1} v & \\ T_{t}=D \Delta T+u-\delta_{2} T & \text { in } \Omega \\ \frac{\partial u}{\partial n}=(1-\xi) H \alpha(x) K(T) & \\ \frac{\partial v}{\partial n}=(1-\xi) H(1-\alpha(x)) K(T) & \\ D \frac{\partial T}{\partial n}=-\sqrt{D \delta_{2}} T & \text { on } \partial \Omega .\end{cases}
$$


The model now only depends on $\alpha(x) \in[0,1], \xi \in[0,1], \delta_{1}, \delta_{2}, D, H>0$. The new values for the parameters can be found in (A.2) in the appendix.

2.1. An intermediate system. We have proposed a PDE model for the inflammatory wound healing phase in order to reproduce the possible non-homogeneous aspects of this process. However, it does not contain the solutions of the ODE (1.1). To extend the solution of the ODE related to (2.6), we will propose an homotopy in the equation in such a way that we can begin in (2.6) and end up with a PDE that contains the solutions of the ODE. In this way we will have a starting point to do our analysis and numerics. This is the reason why we propose the following intermediate system:

$$
\begin{cases}u_{t}=D_{1} \Delta u+\xi \alpha(x) K(T)+\beta u(1-\gamma(u+v))-\delta_{1} u & \\ v_{t}=D_{1} \Delta v+\xi(1-\alpha(x)) K(T)+\beta v(1-\gamma(u+v))-\delta_{1} v & \\ T_{t}=D_{2} \Delta T+\eta u-\delta_{2} T & \text { in } \Omega \\ D_{1} \frac{\partial u}{\partial n}=(1-\xi) H \alpha(x) K(T) & \\ D_{1} \frac{\partial v}{\partial n}=(1-\xi) H(1-\alpha(x)) K(T) & \text { on } \partial \Omega \\ D_{2} \frac{\partial T}{\partial n}=\varepsilon \sqrt{\frac{D_{2}}{\delta_{2}}} \eta u-\sqrt{D_{2} \delta_{2}} T & \end{cases}
$$

whose non-dimensional version is:

$$
\begin{cases}u_{t}=\Delta u+\xi \alpha(x) K(T)+u(1-(u+v))-\delta_{1} u & \\ v_{t}=\Delta v+\xi(1-\alpha(x)) K(T)+v(1-(u+v))-\delta_{1} v & \\ T_{t}=D \Delta T+u-\delta_{2} T & \text { in } \Omega \\ \frac{\partial u}{\partial n}=(1-\xi) H \alpha(x) K(T) & \\ \frac{\partial v}{\partial n}=(1-\xi) H(1-\alpha(x)) K(T) & \\ D \frac{\partial T}{\partial n}=\varepsilon \sqrt{\frac{D}{\delta_{2}}} u-\sqrt{D \delta_{2}} T & \text { on } \partial \Omega\end{cases}
$$

(remember that, as in (2.6), we have denoted without ${ }^{*}$ all the nondimensional functions and parameters, for simplicity). The new parameter $\varepsilon \in[0,1]$ is simply a homotopy parameter that connects both points of view. That is, when $\varepsilon=0$ the $\operatorname{PDE}(2.7)$ is simply the previously proposed PDE model (2.1), while when $\varepsilon=1$ the PDE (2.7) is a system 
that includes the ODE (1.1) as a particular case. Indeed, observe that for $\varepsilon=1, \xi=1$, and $\alpha(x)=\alpha$ the homogeneous solutions of $(2.7)$ are the same as the solutions of the ODE (1.1). In particular, for these values of the previous parameters the constant equilibria of (2.7) are the equilibria of the ODE (1.1). It is in this sense that we say that the system (2.7) generalizes the ODE approach when $\varepsilon=1$.

In Sections 3 and 4 the analytical and numerical study will be done for this intermediate system. So we will be doing the analysis for all the cases of $\varepsilon \in[0,1]$ (observe that this includes the case $\varepsilon=0$, that is the PDE model (2.1)). Also, the fact that we can recover the results for the ODE given in [15] when $\varepsilon=\xi=1$ and $\alpha(x)=\alpha$ in the intermediate approach, will be used to validate the numerical methods that we apply. We will also see that some more results for this case will be obtained using this intermediate system.

With this, we get a well posed system that extends the original ODE model. The next step would be to study the geometry of the wounds and its relationship with diabetes, which will be addressed in future works.

\section{Analytical results}

The aim of the present section is to show that (2.8) is a well-posed problem that generalizes the ODE approach proposed in [15]. This will be stated in Theorem 3.11 below. Let us remark that the well-posedness of (2.8) will be also true for $\varepsilon=0$, which means that (2.6) (or (2.1)) will be a well-posed problem too.

Let us begin with some notation and background results on the functional setting, that can be found, for instance, in [1] and [5]. Consider $\Omega$ a bounded domain of $\mathbb{R}^{n}(n=1,2)$, with its boundary $\partial \Omega$ of class $C^{2}$. Let us write $\mathcal{H}^{1}(\Omega):=H^{1}(\Omega) \times H^{1}(\Omega) \times H^{1}(\Omega)$, where $H^{1}(\Omega)$ is the usual Sobolev space of real $L^{2}(\Omega)$ functions with derivative also in $L^{2}(\Omega)$. The same notation holds for $\mathcal{H}^{s}(\Omega)$ for $s \in \mathbb{R}, \mathcal{L}^{p}(\Omega)$ for $1 \leq p \leq \infty, \mathcal{C}(\Omega)$ and so on.

Let $A: D(A) \subset \mathcal{L}^{2}(\Omega) \longrightarrow \mathcal{L}^{2}(\Omega)$ be the operator $A=\operatorname{diag}\left(A_{1}, A_{2}, A_{3}\right)$ defined by $D(A)=D\left(A_{1}\right) \times D\left(A_{2}\right) \times D\left(A_{3}\right)$ with

$$
\begin{aligned}
& D\left(A_{i}\right)=\left\{\phi \in H^{2}(\Omega) ; B_{i} \phi \equiv a_{i} \frac{\partial \phi}{\partial n_{i}}+b_{i} \phi=0 \text { on } \partial \Omega\right\} \\
& A_{i} \phi=-a_{i} \Delta \phi+c_{i} \phi, \quad \phi \in D\left(A_{i}\right), \quad i=1,2,3
\end{aligned}
$$

with $c_{1}=c_{2}=\delta_{1}-1$ and $c_{3}=\delta_{2}$, which are all positive numbers, $a_{1}=$ $a_{2}=1$ and $a_{3}=D>0, b_{1}=b_{2}=0$ and $b_{3}=\sqrt{D \delta_{2}}$ and $\partial / \partial n_{i}$ denoting 
the outward normal derivative to $\partial \Omega$ associated to the operator $A_{i}$. As in [1], we recall that $A$ is a sectorial operator in $X=\mathcal{L}^{2}(\Omega)$ and that $X^{s}=X_{1}^{s} \times X_{2}^{s} \times X_{3}^{s}$. Also, $-A$ generates an analytic semigroup on $X^{s}$ that satisfies the following estimates:

$$
\begin{aligned}
& \left\|\mathrm{e}^{-\mathrm{At}} \mathrm{u}_{0}\right\|_{X^{s}} \leq M \mathrm{e}^{-\mathrm{rt}}\left\|\mathrm{u}_{0}\right\|_{\mathrm{X}^{\mathrm{s}}}, \quad t \geq 0 \\
& \left\|\mathrm{e}^{-\mathrm{At}} \mathrm{u}_{0}\right\|_{X^{s}} \leq M \mathrm{e}^{-\mathrm{rt}} \mathrm{t}^{-\mathrm{s}}\left\|\mathrm{u}_{0}\right\|_{\mathrm{X}}, \quad t>0
\end{aligned}
$$

for some $r>0, M \geq 1$. It is also known that $A$ can be extended in a unique way to an operator between $\mathcal{H}^{1}(\Omega)$ and its dual, $\mathcal{H}^{-1}(\Omega)$, where each $A_{i}$ is defined by the bilinear form

$$
\begin{array}{r}
\left\langle A_{i}(u), \phi\right\rangle=\int_{\Omega} a_{i} \nabla u \nabla \phi+c_{i} \int_{\Omega} u \phi+b_{i} \int_{\partial \Omega} u \phi, \text { for } u, \phi \in H^{1}(\Omega), \\
i=1,2,3 .
\end{array}
$$

The extended operator $A$ is also sectorial and the analytic semigroup generated by $-A$ also verifies estimates (3.1) and (3.2).

From now on, let $\Omega$ denote the wound site. Observe that $\Omega$ is a bounded domain of $\mathbb{R}^{n}(n=1,2)$ with its boundary $\partial \Omega$ of class $C^{2}$. Now writing $U=(u, v, T)$, Problem (2.8) can be written in a more abstract form (now including the initial conditions explicitly):

$$
\begin{cases}\frac{d U}{d t}+A U=F(x, U) & \text { in } \Omega \\ B U=G(x, U) & \text { on } \partial \Omega \\ U(0)=U_{0}, & \end{cases}
$$

where

$$
\begin{aligned}
& A U=\left(-\Delta u+c_{1} u,-\Delta v+c_{2} v,-D \Delta T+c_{3} T\right) \\
& B U=\left(\frac{\partial u}{\partial n}, \frac{\partial v}{\partial n}, D \frac{\partial T}{\partial n}+b_{3} T\right)
\end{aligned}
$$

(with $\partial / \partial n$ representing the outward normal derivative associated with the previous elliptic operator in $\Omega)$ and $F=\left(f_{1}, f_{2}, f_{3}\right): \Omega \times \mathbb{R}^{3} \longrightarrow \mathbb{R}^{3}$ 
and $G=\left(g_{1}, g_{2}, g_{3}\right): \partial \Omega \times \mathbb{R}^{3} \longrightarrow \mathbb{R}^{3}$ being the following nonlinearities

$$
\begin{aligned}
& f_{1}(x, U)=\xi \alpha(x) K(T)-u(u+v) \\
& f_{2}(x, U)=\xi(1-\alpha(x)) K(T)-v(u+v) \\
& f_{3}(x, U)=u \\
& g_{1}(x, U)=(1-\xi) H \alpha(x) K(T) \\
& g_{2}(x, U)=(1-\xi) H(1-\alpha(x)) K(T) \\
& g_{3}(x, U)=\varepsilon \sqrt{\frac{D}{\delta_{2}}} u .
\end{aligned}
$$

The following lemma is an immediate consequence of the Sobolev embeddings.

Lemma 3.1. Assume that the function $K$ is smooth enough and satisfies that $K(s)>0$ in $\left[0, T_{Q}\right.$ ) (for a certain $T_{Q}>0$ ), $K\left(T_{Q}\right)=0$, is extended by zero outside, and has a maximum $\bar{K}$ in $\left(0, T_{Q}\right)$. Assume also that $\alpha(x) \in[0,1]$ is $C^{2}(\Omega)$. Define $L: \mathcal{H}^{1}(\Omega) \longrightarrow \mathcal{H}^{-1}(\Omega)$ as

$$
\langle L(U), \Phi\rangle=\langle F(x, U), \Phi\rangle_{\Omega}+\langle G(x, U), \Phi\rangle_{\partial \Omega}, \quad U, \Phi \in \mathcal{H}^{1}(\Omega),
$$

where $\langle,\rangle_{\Omega},\langle,\rangle_{\partial \Omega}$ indicate respectively the $\mathcal{L}^{2}(\Omega)$ and $\mathcal{L}^{2}(\partial \Omega)$ inner products. Then, $L$ is continuous and locally Lipschitz continuous in $\mathcal{H}^{1}(\Omega)$.

Remark 3.2. When solving problems involving non-homogeneous boundary conditions, it is natural to consider them as in (3.5). As we will see below, this allows to incorporate the boundary conditions as part of the equation in the weak formulation of the problem.

Let us also remark that in the definition in (3.5) we are omitting the reference to the trace operator $\gamma: \mathcal{H}^{1}(\Omega) \rightarrow \mathcal{H}^{\frac{1}{2}}(\partial \Omega)$.

After Lemma 3.1 we can associate with Problem (3.3) its dual formulation in $\mathcal{H}^{-1}(\Omega)$ as usual:

$$
\left\langle\frac{d U}{d t}, \Phi\right\rangle=-\langle A(U), \Phi\rangle+\langle L(U), \Phi\rangle, \quad U, \Phi \in \mathcal{H}^{1}(\Omega),
$$

where $A$ is defined as in (3.3) and $L$ as in (3.5).

Remark 3.3. With the above assumptions and following [4] (see Corollary 3.1 of [1] ) one can get that (3.6) has a local solution

$$
U \in \mathcal{C}\left(\left[0, t_{\max }\right), \mathcal{H}^{1}(\Omega) \cap \mathcal{C}(\Omega)\right) \cap \mathcal{C}\left(\left(0, t_{\max }\right), \mathcal{H}^{s}(\Omega)\right)
$$


and

$$
\frac{d U}{d t} \in \mathcal{C}\left(\left(0, t_{\max }\right), \mathcal{H}^{s}(\Omega)\right)
$$

for $0<s<\frac{3}{2}$ and $t_{\max }>0$. However, since we also want to show the existence of a positively invariant set, we will use the approach of [5], although it also gets local existence.

In order to state the invariance and existence results, some notations and definitions are needed.

Definition 3.4 (from $[5]$ ). We say that $U:\left[0, t_{\max }\right) \rightarrow \mathcal{L}^{2}(\Omega)$, with $t_{\max }>0$, is a weak solution of Problem (3.3) if

(a) $U$ is continuous on $\left[0, t_{\max }\right)$ and $U(0)=U_{0}$.

(b) $U$ is absolutely continuous on compact subsets of $\left(0, t_{\max }\right)$.

(c) $U$ is differentiable almost everywhere on $\left(0, t_{\max }\right)$ and, for each $t$ where the derivative exists, $U$ satisfies (3.6).

We say that $U$ is a global (weak) solution if $t_{\max }=\infty$.

Definition 3.5 (from $[6]$ ). We say that $U:[0, \infty) \times \bar{\Omega} \rightarrow \mathbb{R}^{3}$ is a global classical solution if $U$ is $C^{1}$ in time, $C^{2}$ in space and satisfies (3.3) for all $(t, x) \in[0, \infty) \times \bar{\Omega}$.

Definition 3.6 (from [5]). A subset $\mathcal{S} \subset \mathcal{L}^{2}(\Omega)$ is called positively invariant if for any $U$ weak solution of Problem (3.3) with $U(0) \in \mathcal{S}$ then $U(t) \in \mathcal{S}$ for all $t \in\left(0, t_{\max }\right)$.

Definition 3.7. Let $\mu_{1}$ be the first eigenvalue (associated with the eigenfunction $\phi_{1}$ ) of the Stekloff problem

$$
\begin{cases}-\Delta \phi+\left(\delta_{1}-1\right) \phi=0 & \text { in } \Omega \\ \frac{\partial \phi}{\partial n}=\mu \phi & \text { on } \partial \Omega .\end{cases}
$$

Definition 3.8. Let $\lambda_{1}$ be the first eigenvalue (associated with the eigenfunction $\psi_{1}$ ) of the eigenvalue problem

$$
\begin{cases}-D \Delta \psi=\lambda \psi & \text { in } \Omega \\ D \frac{\partial \psi}{\partial n}+\sqrt{D \delta_{2}} \psi=0 & \text { on } \partial \Omega .\end{cases}
$$


Remark 3.9. Following [10], we get that $\mu_{1}, \lambda_{1}, \phi$, and $\psi_{1}$ are strictly positive.

Definition 3.10. Let us consider $M_{1}, M_{2}>0$ such that

$$
M_{1} \geq \frac{\bar{K}}{\delta_{1}-1}, \quad M_{2} \geq \frac{H \bar{K}}{\mu_{1} \min \phi_{1}}
$$

and define $\bar{u}=\bar{v}=M_{2} \phi_{1}+M_{1}$, where $\bar{K}$ is defined as in Lemma 3.1. With $\bar{u}$, consider $N_{1}, N_{2}>0$ such that

$$
N_{1} \geq \frac{\varepsilon}{\delta_{2}} \max \bar{u}, \quad N_{2} \geq \frac{\max \bar{u}}{\left(\lambda_{1}+\delta_{2}\right) \min \psi_{1}}
$$

and define $\bar{T}=N_{2} \psi_{1}+N_{1}$.

Following the notation used in [5], let us also define $\Phi=(0,0,0)$, $\Psi=(\bar{u}, \bar{v}, \bar{T})$, and $\operatorname{set} Q$ as

(3.11) $Q:=[\Phi, \Psi]=\left\{(u, v, T) \in \mathcal{L}^{2}(\Omega) ;(0,0,0) \leq(u, v, T) \leq(\bar{u}, \bar{v}, \bar{T})\right.$ a.e. $\}$.

Theorem 3.11 (Global existence). Assume that the function $K$ and $\alpha(x)$ are as in Lemma 3.1 and that the parameters $H, \delta_{1}, \delta_{2}, \xi$ in (3.4) are such that

$$
0 \leq \xi \leq 1, \quad \delta_{1}>1, \quad \varepsilon \geq 0, \quad H, \delta_{2}>0
$$

Then,

1. $Q$ defined in (3.11) is a positively invariant set for (3.3).

2. For any $U_{0} \in Q$ there exists a unique global weak solution $U \in Q$ for $(3.3)$.

3. For $t>0$, if $U_{0} \in Q \cap \mathcal{C}(\bar{\Omega})$, then $U(t)$ is a global classical solution for $(3.3)$.

Proof: The first step of the proof is a direct application of Theorem 1 of [5], where one uses sub and super solutions to prove part 2 of the present theorem. More concretely, for this part one just need Lemma 3.1 and to verify part 1 . Once we have existence of weak solutions, we can use a bootstrap argument to prove part 3 .

Let us begin with the proof of part 1. First, from the regularity and positiveness of the eigenfunctions $\phi_{1}$ and $\psi_{1}$ given in Definitions 3.7 and 3.8, we have that $\Phi, \Psi \in \mathcal{H}^{1}(\Omega)$ and $\Phi \leq \Psi$. To prove that $Q$ is a positively invariant set for Equation (3.3) we need to check the following 
inequalities:

$$
\begin{array}{ll}
0 \leq f_{1}(x,(0, v, T)), & 0 \leq g_{1}(x,(0, v, T)), \\
0 \leq f_{2}(x,(u, 0, T)), & 0 \leq g_{2}(x,(u, 0, T)), \\
0 \leq f_{3}(x,(u, v, 0)), & 0 \leq g_{3}(x,(u, v, 0))
\end{array}
$$

and

$$
\begin{array}{ll}
A_{1} \bar{u} \geq f_{1}(x,(\bar{u}, v, T)), & B_{1} \bar{u} \geq g_{1}(x,(\bar{u}, v, T)), \\
A_{2} \bar{v} \geq f_{2}(x,(u, \bar{v}, T)), & B_{2} \bar{v} \geq g_{2}(x,(u, \bar{v}, T)), \\
A_{3} \bar{T} \geq f_{3}(x,(u, v, \bar{T})), & B_{3} \bar{T} \geq g_{3}(x,(u, v, \bar{T})),
\end{array}
$$

whenever $\Phi \leq(u, v, T) \leq \Psi$ (see (3.4) for recalling the definitions of these functions and (3.11) for recalling the definition of $Q$ ). The proof of the lower bounds (3.12) is immediate from the positiveness of all the terms. As we are going to see, the proof of the upper ones follows straightforward from the definition of $Q$.

Thus, we get that for the first face

$$
A_{1} \bar{u}=\left(-\Delta+c_{1}\right)\left(M_{2} \phi_{1}+M_{1}\right)=\left(\delta_{1}-1\right) M_{1} \geq \bar{K} \geq f_{1}(x,(\bar{u}, v, T))
$$

and

$$
B_{1} \bar{u}=\frac{\partial}{\partial n}\left(M_{2} \phi_{1}+M_{1}\right)=M_{2} \mu_{1} \phi_{1} \geq H \bar{K} \geq g_{1}(x,(\bar{u}, v, T)) .
$$

This proves the inequality for the first face. For the second one it can be seen in a similar way. For the third face

$$
\begin{aligned}
A_{3} \bar{T} & =\left(-D \Delta+c_{3}\right)\left(N_{2} \psi_{1}+N_{1}\right)=N_{2}\left(\lambda_{1}+\delta_{2}\right) \psi_{1}+\delta_{2} N_{1} \\
& \geq N_{2}\left(\lambda_{1}+\delta_{2}\right) \min \psi_{1} \geq \max \bar{u} \geq f_{3}(x,(u, v, \bar{T}))
\end{aligned}
$$

and

$$
\begin{aligned}
B_{3} \bar{T} & =\left(D \frac{\partial}{\partial n}+\sqrt{D \delta_{2}}\right)\left(N_{2} \psi_{1}+N_{1}\right)=\sqrt{D \delta_{2}} N_{1} \\
& \geq \varepsilon \sqrt{\frac{D}{\delta_{2}}} \max \bar{u} \geq g_{3}(x,(u, v, \bar{T})) .
\end{aligned}
$$

Observe that $Q$ is a bounded subset in $\mathcal{L}^{\infty}(\Omega)$. Hence, Theorem 1 of [5] applies and gives that $Q$ is a positively invariant set and also the existence and uniqueness of a global weak solution.

Let us now prove part 3. By Remark 3.3 we know that

$$
U(t), \quad \frac{d U(t)}{d t} \in \mathcal{H}^{s}(\Omega), \quad 0<s<\frac{3}{2}
$$


so, from the embeddings of the spaces, we have

$$
\frac{d U(t)}{d t} \in \mathcal{C}^{\delta}(\bar{\Omega}), \quad \delta<\frac{1}{2}
$$

We want to show that $U(t, \cdot)$ is, in fact, $\mathcal{C}^{2}(\bar{\Omega})$ for each $t>0$ and that satisfies (3.3), that is, we want to show that it is a global classical solution.

We now denote $\left(u_{1}, u_{2}, u_{3}\right)=(u, v, T)$ and fix $u_{j}$ if $j \neq i$ in each component of $f_{i}, g_{i}, i=1,2,3$. That is, we consider the following functions

$$
\begin{gathered}
\tilde{f}_{1}(t, x, z)=f_{1}\left(x, z, u_{2}(t, x), u_{3}(t, x)\right) \\
\tilde{f}_{2}(t, x, z)=f_{2}\left(x, u_{1}(t, x), z, u_{3}(t, x)\right) \\
\tilde{f}_{3}(t, x, z)=f_{3}\left(x, u_{1}(t, x), u_{2}(t, x), z\right)
\end{gathered}
$$

and, similarly, $\tilde{g}_{1}, \tilde{g}_{2}$, and $\tilde{g}_{3}$. Then, from the regularity of $f_{i}, g_{i}$ for $i=1,2,3$ we get

$$
\tilde{f}_{i}\left(t, \cdot u_{i}(t, \cdot)\right)-\frac{d u_{i}(t, \cdot)}{d t} \in L^{2}(\Omega), \quad \tilde{g}_{i}\left(t, \cdot, u_{i}(t, \cdot)\right) \in H^{1}(\Omega)
$$

and thus, from the trace theorem,

$$
\gamma\left(\tilde{g}_{i}\left(t, \cdot u_{i}(t, \cdot)\right)\right) \in \mathcal{H}^{\frac{1}{2}}(\partial \Omega),
$$

where $\gamma$ denotes the corresponding trace operator. Fixing the solution $u_{i}$ obtained from part 2 and $t>0$, we can consider the elliptic problems

$$
\left\{\begin{array}{l}
A_{i} z=\tilde{f}_{i}\left(t, x, u_{i}(t, x)\right)-\frac{d u_{i}(t, x)}{d t} \\
\frac{d z}{d n_{i}}=\tilde{g}_{i}\left(t, x, u_{i}(t, x)\right), \quad i=1,2,3
\end{array}\right.
$$

We can apply the elliptic regularity results (see $[7]$ ) to conclude that $z_{i}$, the solution of $(3.14)$, is in $H^{2}(\Omega)$.

Now we want to show that $z_{i}=u_{i}(t, \cdot)$. From Green's formula, it follows that for all $w_{i} \in H^{2}(\Omega)$ and for all $\varphi \in H^{2}(\Omega)$ with $B_{i} \varphi=0$ we get:

$$
\begin{aligned}
\int_{\Omega}\left(A_{i} w_{i}\right) \varphi-\int_{\Omega} w_{i}\left(A_{i} \varphi\right) & =-a_{i} \int_{\partial \Omega} \frac{\partial w_{i}}{\partial n} \gamma(\varphi)+a_{i} \int_{\partial \Omega} \gamma\left(w_{i}\right) \frac{\partial \varphi}{\partial n} \\
& =-\int_{\partial \Omega}\left(a_{i} \frac{\partial w_{i}}{\partial n}+b_{i} \gamma(w)\right) \gamma(\varphi)
\end{aligned}
$$


where $\gamma$ stands for the corresponding trace operator. Applying (3.15) to $z_{i}$, solution of $(3.14)$, it follows that

$$
\begin{array}{r}
\int_{\Omega}\left(\tilde{f}_{i}\left(t, x, u_{i}(t, x)\right)-\frac{d u_{i}(t, x)}{d t}\right) \varphi(x) d x-\int_{\Omega} z_{i}(x)\left(A_{i} \varphi(x)\right) d x \\
=-\int_{\partial \Omega} \gamma\left(\tilde{g}_{i}\left(t, x, u_{i}(t, x)\right)\right) \gamma(\varphi(x)) d x .
\end{array}
$$

Thus, $z_{i}$ satisfies (3.14) in $H^{-1}(\Omega)$. But as $u_{i}$ is the unique solution in $H^{-1}(\Omega)$ we can conclude that $u_{i}=z_{i} \in H^{2}(\Omega)$. Applying the embedding results once more, we get $u_{i}(t, \cdot) \in C^{1}(\bar{\Omega}), i=1,2,3$, and applying the Classical Existence Theorem (see [6, p. 128]) we conclude that $u_{i}(t, \cdot) \in C^{2}(\bar{\Omega}), i=1,2,3$. Hence, $U(t, x)$ is a classical solution of $(3.3)$.

Remark 3.12. According to the values of the parameters given in the appendix (coming from [15]), the set $Q$ defined in (3.11) is non-empty. Observe also that the solutions in the invariant set are positive and bounded. So, they are acceptable from a biological point of view.

Remark 3.13. Using the same result of [5] but now for the elliptic version of the problem, we can assure the existence of equilibrium solutions in the invariant region $Q$. Concerning the question whether they are constant or not, a simple calculation shows that this depends on the values of the parameters. More concretely, when $\varepsilon=1, \xi=1$, and $\alpha(x) \equiv \alpha$ is constant there exist constant equilibria in $Q$ and it can be easily seen that these are the same as the equilibria of the ODE system (1.1). In this sense, we say that the proposed PDE model (2.1) generalizes the ODE approach given in [15]. However, if $\varepsilon=1$ but $\xi \neq 1$ it can be seen that there do not exist constant equilibria, even for $\alpha(x)$ being constant, except for a very specific value of $\alpha=\alpha^{*}$ (that, for our values of the parameters given in the appendix, is $\left.\alpha^{*}=0.588421\right)$. Finally, when $\varepsilon \neq$ 1 , there only exist constant equilibria if $\xi=1$ and $\alpha=0$. In this case, the equilibrium solution is of the form $\left(0, v^{*}, 0\right)$, which in non-dimensional parameters is explicitly given by $v^{*}=\frac{1}{2}\left(1-\delta_{1}+\sqrt{1-\delta_{1}+4 K(0)}\right)$. In particular, these results show that with the PDE system we obtain profiles that are non-constant in space, which was not possible with an ODE approach. Hence, the ODE model does not suffice.

\section{Numerical results}

In this section we focus on the existence of stable equilibrium solutions for (2.7), the dimensional version of the intermediate PDE problem, in 
the particular case that $\alpha$ is constant. First we treat the one-dimensional space domain and compare the results in this case with those of the ODE approximation (1.1). In this case we can make some bifurcation figures fixing all the parameters and moving either $\alpha, \xi$ or $\varepsilon$. Finally, we show some results for a two-dimensional space square domain, for the same set of parameters. An accurate bifurcation study is left for a future work.

All the computations in this section are going to be done using the values of the fixed parameters in (A.1) in the appendix (given by [15]).

\subsection{Bifurcation branches of equilibria for the one-dimensional} case. Let us recall that the equilibria of the ODE model (1.1) are constant equilibria for Problem (2.7) when $\xi=1, \varepsilon=1$ and all constant $\alpha$, but not for $\xi \neq 1$ nor $\varepsilon \neq 1$. Since we have continuity of equilibria, there must exist a branch of equilibrium solutions coming from each equilibrium of the ODE. Each one of these solutions are related with the possibility or not of a correct healing of the wound.

Let us also remember that, from the results in the ODE model, we take two biological relevant values for $\alpha$, namely $\alpha_{\text {healthy }}=0.5$ and $\alpha_{\text {diabetic }}=0.8$ (see Section 1 ) and that for $\xi$ and $\varepsilon$ the relevant values are 0 and 1 (see Section 2). We are going to use these values as references to do the numerics. The goal is to show branches of stable equilibria as one of the values of the parameters $\xi, \alpha$ or $\varepsilon$ moves.

First, we fix $\varepsilon=1$ and draw the branches fixing either $\alpha$ or $\xi$ and moving the other one. Since, at least, we want to capture the same number of equilibria as the ODE, this has to be done in a specific order.

In Figure 4.1 we show the way we fix and move the parameters. We take starting points $\bullet$ in the parameter space and do four continuations (1), (2), (3), and (4). First, starting at $\xi=1$ and $\alpha=0.8$, we fix $\alpha=0.8$ and we continue the three constant equilibria of the ODE, which are also constant equilibria for (2.7) when $\xi=1$, moving $\xi$ from 1 to 0 (this corresponds to branch (1) of the continuation shown in Figure 4.1) and from 1 to 2 . Then fixing $\xi=1$ we move $\alpha$ from 0.8 to 1 and from 0.8 to 0 (branch (2)). After that, we start at $\xi=0$ and $\alpha=0.5$, taking as initial conditions values close to the three constant equilibria of the ODE. We fix $\xi=0$ and move $\alpha$ from 0.5 to 1 and from 0.5 to 0 (corresponding to branch (3) of the continuation). Finally, fixing $\alpha=0.5$ we move $\xi$ from 0 to 2 (branch (4)).

Although in our model $\xi \in[0,1]$, we continue this parameter between 1 and 2 in order to show how the branches of solutions connect. We observe that, in all cases, we get saddle node bifurcations, giving rise to a hysteresis phenomenon. 


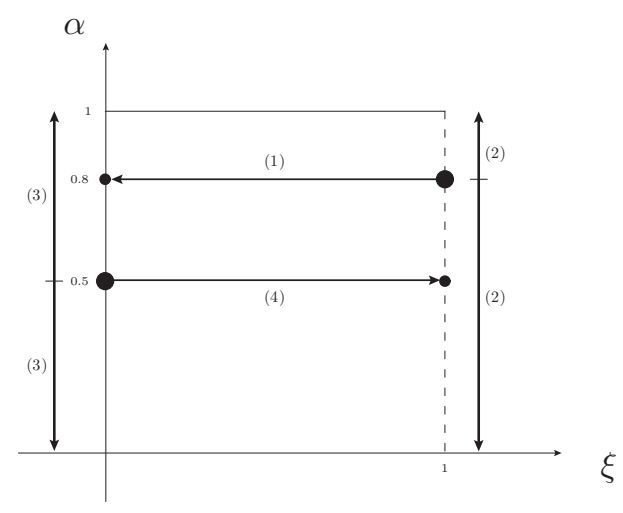

FIgURE 4.1. Schematic in parameter space of the continuations of equilibria. The numbers in parenthesis refer to each branch in the order that they are done. The large dots are the starting points. The dashed line are the parameters where the ODE is a solution.

We have a parabolic one dimensional partial differential system of equations with Neumann boundary conditions, which can be homogeneous for $u$ and $v$ when $\xi=1$. For the integration of the system we follow a standard procedure, integrating in time the partial differential system by directly using a solver given by Matlab. In all of our numerical computations we consider the known equilibria that we obtain when we fix the pair of parameters $\xi$ and $\alpha$ as the initial conditions for the integration and we continue each one moving one of the parameters in the way we mentioned before.

In Figure 4.2 we show the branches in $\xi$ of the $L^{2}$ norm of the solutions $u, v$, and $T$ for $\alpha=0.8$ for different initial conditions (represented by different colors in the figure). We observe that for certain $\xi_{1}, \xi_{2}>0$, when $0<\xi<\xi_{1}$ all initial conditions go to the equilibrium with larger norm (the non-healed state), when $\xi_{2}<\xi<2$ all of them go to the equilibrium with smaller norm (the healed state) and for the values in between there must be three different equilibria, unless for two values of $\xi$ with exactly two solutions. The unstable state is not seen in the previous figure because of the numerical method used for computing the equilibria. Therefore, for these intermediate values of $\xi$, achieving a healed or a non-healed state will depend on the initial conditions. We can see that the behaviour is the same for inflammatory and repair macrophage 
densities and for the TGF- $\beta$ concentration, and all of them present this hysteresis phenomenon. This can be assured since for $\xi=1$ the equilibria are constant and its stability is known from the results in [15]. In the numerics those are the only values of $\alpha$ and $\xi$ where the unstable equilibria can be seen (see Figure 4.2).
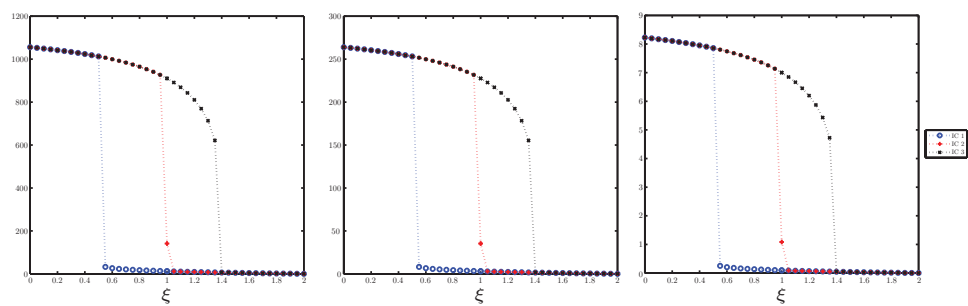

Figure 4.2. From left to right, continuation of the equilibria $u, v$, and $T$ in $\xi$ for $\alpha=0.8$ and different initial conditions (branch (1)).

In Figure 4.3 we show the branches in $\xi$ of the $L^{2}$ norm of the solutions $u, v$, and $T$ for $\alpha=0.5$ for different initial conditions (represented by different colors in the figure). We observe a similar behaviour as before for the branches of solutions in this case, but with different values for $\xi_{1}$ and $\xi_{2}$. In particular, we can observe that $\xi_{2}<1$ when $\alpha=0.5$. This makes that, for values of $\xi$ close to 1 , the only possible state for the non-diabetic wounds is the healed one, while for the diabetic ones both the healed and non-healed states are possible as $\xi_{2}>1$ when $\alpha=0.8$.
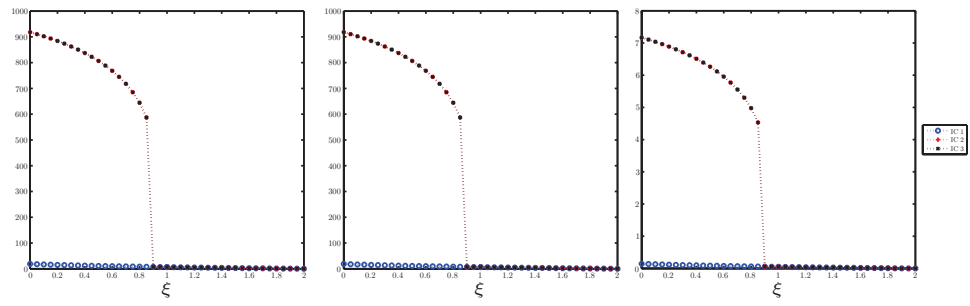

Figure 4.3. From left to right, continuation of the equilibria $u, v$, and $T$ in $\xi$ for $\alpha=0.5$ and different initial conditions (branch (4)).

In Figures 4.4 and 4.5 we show the branches in $\alpha$ of the $L^{2}$ norm of the solutions $u, v$, and $T$ for $\xi=0$ and $\xi=1$, respectively, also for 
different initial conditions (represented by different colors in the figure). Now, for certain $\alpha_{1}, \alpha_{2}>0$, we observe that when $0<\alpha<\alpha_{1}$ all initial conditions go to the equilibrium with smaller norm (the healed state), when $\alpha_{2}<\alpha<1$ all of them go to the equilibrium with larger norm (the non-healed state) and for the values in between we have three different equilibria, unless for two values of $\alpha$ with exactly two solutions. Again, the unstable state is not seen in the previous figure because of the numerical method used for computing the equilibria, except in Figure 4.5 when $\alpha=0.8$ (as $\xi=1$, this is in agreement with the ODE results). Also, in the situation of these intermediate values of $\alpha$ the healing of the wound will depend on the starting conditions. The behaviour is the same for inflammatory and repair macrophage densities and for the TGF- $\beta$ concentration. In theses cases we also have a hysteresis phenomenon with a mirrored bifurcation diagram (compared to the $\xi$ continuation one).
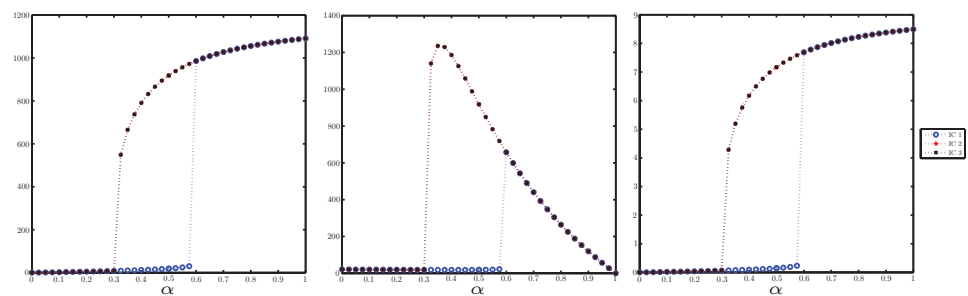

Figure 4.4. From left to right, continuation of the equilibria $u, v$, and $T$ in $\alpha$ for $\xi=0$ and different initial conditions (branch (3)).
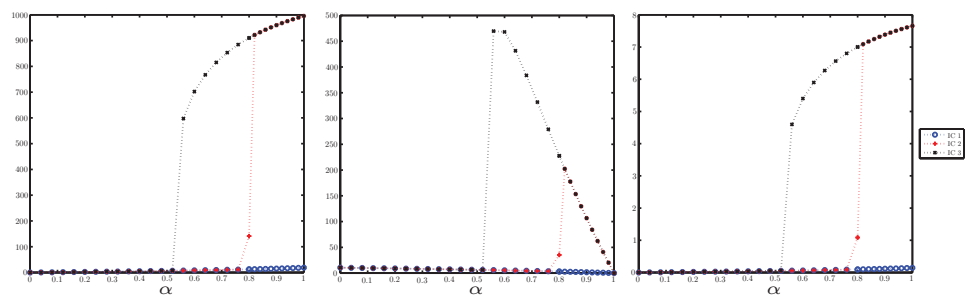

Figure 4.5. From left to right, continuation of the equilibria $u, v$, and $T$ in $\alpha$ for $\xi=1$ and different initial conditions (branch (2)). 
Finally Figure 4.6 shows the profiles of the equilibria for $u$ when $\alpha=$ 0.8 and for $\xi=0,0.1,0.2, \ldots, 1$. The left figure shows the hysteresis phenomenon mentioned above. In this way, we see that for $\xi>\xi_{2}$ they converge to the healed states and for $\xi<\xi_{1}$ they converge to the nonhealed ones. The right figure shows the equilibria corresponding to initial conditions converging only to the non-healed states. Observe that they vary continuously with $\xi$ and get more nonconstant as $\xi$ tends to 0 .
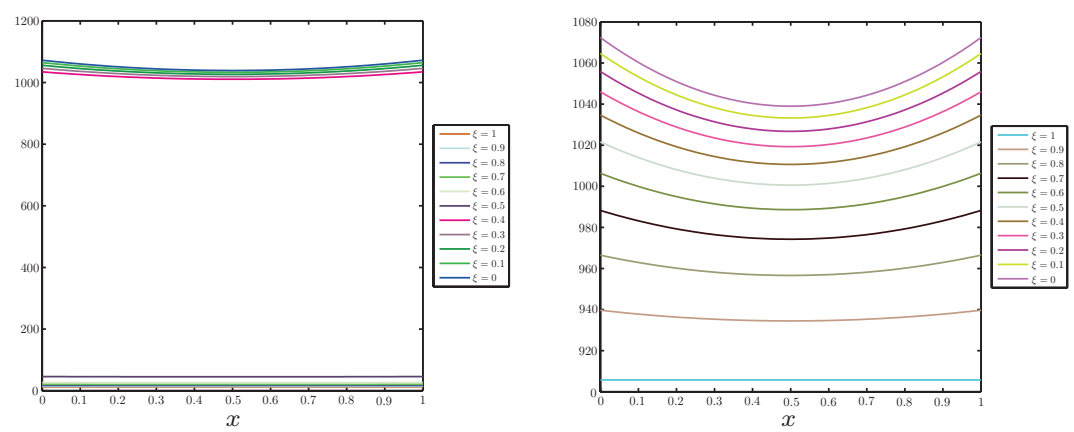

FIGURE 4.6. Stable state profiles of $u$ and hysteresis (left) and the non-healed equilibria branch (right) for $\alpha=0.8$ and $\xi$ from 0 to 1 .

So far we have shown the bifurcation schemes and the profiles for different values of the parameters when $\varepsilon=1$. This is the generalization of the ODE model and for them we can compare the results with those of [15], but also give more information because of the space dependence in the PDE model. In some sense we use this to validate our numerical approximations in order to use them in the model (2.1), that is $(2.7)$ when $\varepsilon=0$, where the constant equilibria given by the equilibria of the ODE are not solutions for any value of $\xi$ or $\alpha$.

So, we now continue the parameter $\varepsilon$ from 1 to 0 , taking as initial conditions those solutions found for $\varepsilon=1$ in the four cases: $\xi=0$ and $\alpha=0.5, \xi=0$ and $\alpha=0.8, \xi=1$ and $\alpha=0.5$, and $\xi=1$ and $\alpha=0.8$. We observe as before hysteresis phenomena in the bifurcation diagrams. In Figure 4.7 we show the bifurcation diagram for $\alpha=0.8$ and $\xi=1$, as an illustration, for different initial conditions (represented by different colors in the figure). 

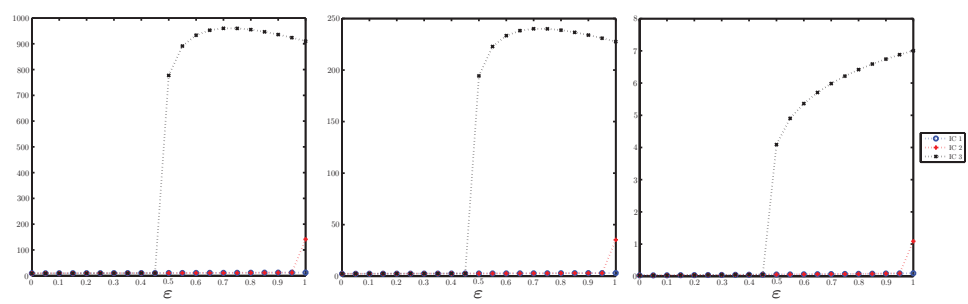

Figure 4.7. From left to right, continuation of the equilibria $u, v$, and $T$ in $\varepsilon$ for $\xi=1, \alpha=0.8$ and different initial conditions.

As before, the saddle-node bifurcation values depend on the values of the parameters and for the values of $D_{2}=\delta_{2}$ used in the previous pictures we can not see the hysteresis phenomenon when $\varepsilon$ tends to zero when $\xi=1$ and $\alpha=0.5$. In order to show this, we consider smaller values for $D_{2}=\delta_{2}$. In Figure 4.8 we show the bifurcation branches for $\xi=1, \alpha=0.5$ and $\delta_{2}=D_{2}=3.5$ and for different initial conditions (represented by different colors in the figure), which represents a smaller diffusion case.
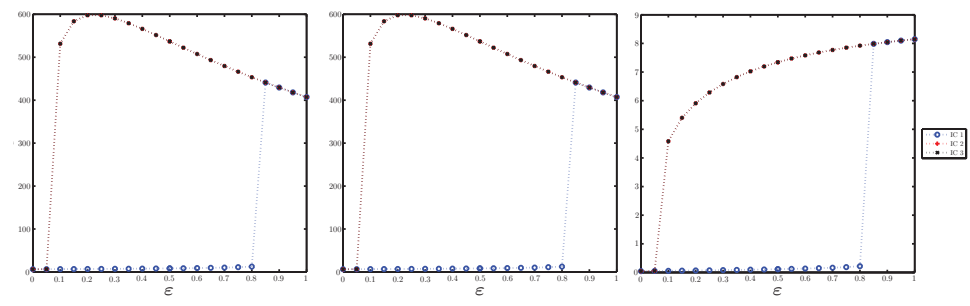

Figure 4.8. From left to right, continuation of the equilibria $u, v$, and $T$ in $\varepsilon$ with small diffusion for $\xi=1$, $\alpha=0.5$ and different initial conditions.

4.2. Some conclusions for the one-dimensional case. In this subsection we are going to give some biological conclusions from the healing point of view related to the values of the parameters $\xi$ and $\alpha$. Let us remember that $\alpha$ corresponds to the diabetes parameter ( 0.5 for nondiabetic and 0.8 for diabetic) and $\xi$ is a parameter that measures the quantity of reaction in the interior and in the boundary ( 0 for reactions only at the boundary and 1 for reactions only in the interior and zero Neumann flux at the boundary). 
When $\varepsilon=1$ and $\xi=1$, System (2.7) is a PDE extension of the ODE model (1.1) and the constant equilibria of (2.7) are also solutions of the ODE. In this case, the bifurcations in $\alpha$ agree with the ODE results seen in [15]. That is, for $\alpha$ small (non-diabetic) we only have the healed equilibrium and for larger $\alpha$ (diabetic) we can heal or not depending on the initial conditions. This results validate the numerical techniques used for the intermediate Problem (2.7). Also, we can now observe that for very large $\alpha$ (close to 1 ) only the non-healed state remains.

When $\varepsilon=1$ and $\xi=0$ the results are qualitatively the same but the values of $\alpha$ where the changes of states occur are smaller than for the corresponding $\alpha$ when $\xi=1$.

But if we now look at the previous numerical results from the point of view of the parameter $\xi$, we can see that the PDE approximation gives additional information to the ODE previous results in two different aspects. The first one is the fact that we now can have equilibrium solutions that are non-constant in space, which cannot happen in an ODE approach. The second one reveals a different behaviour in the healing of solutions from the expected by the ODE model when the parameter $\xi$ is small. More concretely, when $\xi$ is small (that is, the reaction occurs mainly at the boundary) and $\alpha=0.8$ we only have a nonhealed state (which differs from the ODE results when $\alpha=0.8$ ). When $\xi$ increases we keep this non-healed profile, and it also appears a healed one and we only can assure a unique healing state for values of $\xi$ larger than a certain $\xi_{2}>1$, which occurs outside the range of the considered values. On the other hand, for $\alpha=0.5$ we observe the non-healed and healed profiles, depending on the initial conditions, for almost all values of $\xi$ (which also differs from the ODE results when $\alpha=0.5$ ). Only for $\xi$ close to 1 we can assure the correct healing of the wound, which naturally coincides with the conclusions made before for the continuation in $\alpha$ with $\xi=1$. These results show that a PDE approach could be convenient for this healing problem.

From a biological point of view, the following immediate conclusion can be derived from the previous diagrams: either increasing $\alpha$ (more diabetes) or decreasing $\xi$ (TGF- $\beta$ acting mainly at the boundary of the wound site) are situations that difficult the correct healing of the wound.

Finally, Figures 4.7 and 4.8 show how the intermediate system approaches the desired PDE model (that is, $\varepsilon=0$ ). It may seem that the bifurcation branches are lost as $\varepsilon \rightarrow 0$ but, in our opinion, this is not true for the appropriate values of all the parameters involved in the equation. An accurate study of this limit problem is left for a future work, specially in the $2 \mathrm{D}$ case. 
4.3. Some equilibria for the two-dimensional case. In this subsection we are going to show some stable nontrivial and nonconstant equilibrium solutions in the case $\varepsilon=1$ for some values of the parameters in a square domain as well as a bifurcation diagram of the equilibria. We leave for future work a more accurate study of the equilibria and the relation of them with the geometry of the domain, mainly for the case $\varepsilon=0$.

For the computations in this case we consider a discretization of the square and of the equations in the system and compute the solutions directly using Newton method instead of the continuation in time used in the previous subsection. Hence we solve a system $M W=F(W)$ where the matrix $M$ has dimension $3 N$ (where $N$ is the global number of nodes in the discretized domain) and contains the discretization of the Laplacian and part of the linear terms in (2.7). $W=(U, V, T)$ is a $\mathbb{R}^{3 N}$ vector, with $U, V$, and $T$ the vectors of nodal values of the concentrations. And the nonlinear term $F(W)$ contains the nonlinearities of (2.7) and the part of the linear terms not included in $M$. We have to take into account different possibilities for the nonlinearity because choosing a simpler possibility may give a non-convergent method.

In Figure 4.9 we show an example of an stable nonconstant equilibrium solution for $u$ computed when the parameters are $\xi=0$ and $\alpha=0.8$. Observe that, in this situation, we arrive at a non-constant non-healed equilibrium.

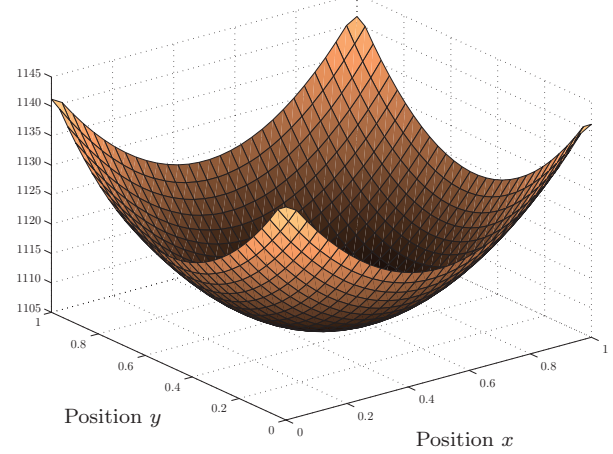

FiguRE 4.9. Equilibrium profile for $u$ when $\xi=0$ and $\alpha=0.8$ (a non-healed one, in this case).

Next, in Figure 4.10, we present the bifurcation diagrams for the first component $u$ of the equilibria in two cases: when $\xi=1$ and varying $\alpha$ 
and for $\alpha=0.8$ and varying $\xi$. Observe that since we are using a fixed point algorithm we can see the unstable branch in the diagrams. Once again we observe saddle node bifurcations and the hysteresis phenomena.
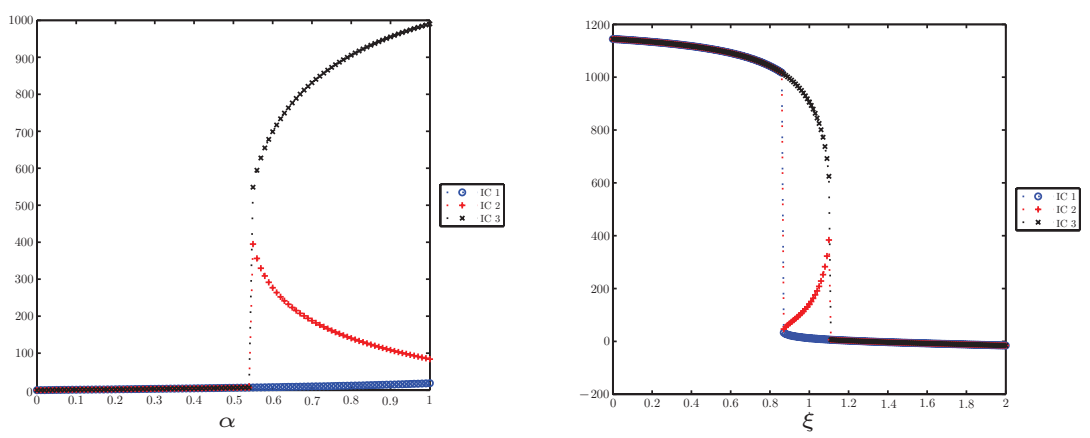

Figure 4.10. Continuation in $\alpha$ when $\xi=1$ (left) and in $\xi$ when $\alpha=0.8$ (right) of equilibria $u$ in the 2D case.

\section{Conclusions}

We have proposed a PDE as a model for the dynamics of the interaction between certain macrophages and a growth factor at the inflammatory phase of wound healing. This model aims at generalizing a previous ODE one. This is done through an intermediate PDE system that can be seen as an homotopy problem connecting both approaches.

Once having shown the well-posedness of the PDE we do some numerical simulations. First, we recover the same results as in the ODE case using the appropriate values of the parameters in the intermediate system and, hence, the numerical scheme is validated. Then, we study the relation between the healing of the wound and different parameters of biological interest obtaining several bifurcation diagrams. We are able to see, for instance, that increasing the diabetic parameter impairs the healing of the wound, as expected. But we can also see that when the income of macrophages due to the growth factor occurs mainly at the boundary of the wound, its healing is also impaired.

As we can see through the paper, the fact of using the PDE model instead of the ODE one allows us to give some additional and important information to the problem. For instance, it allows us to obtain nonconstant equilibrium solutions. Also, when the macrophages income happens mainly through the boundary we obtain a different healing from 
the expected one and the bifurcating parameters are shifted. This also happens when the diffusion coefficients are changed, and this can be related with the domain size. It is clear that all of these facts could not be observed if an ODE model was used.

Acknowledgements. The authors would like to thank Jonathan A. Sherratt for many helpful comments and remarks, mainly in the model and biological conclusions.

\section{Appendix A}

In this appendix we give a list of the values for the parameters involved in the PDE model. First, we give them for the dimensional version (2.7). We have the three non-dimensional parameters $\alpha(x), \xi$, and $\varepsilon$ as well, all of them in $[0,1]$. Also, we have $H=1 \mathrm{~mm}^{-1}$ which is just a boundary dimensional adjustment parameter. The values for the rest of parameters are taken from $[\mathbf{1 5}],[\mathbf{1 6}]$ and the references therein.

(A.1) $\quad \beta=0.03465 \frac{1}{\text { day }} \quad$ (macrophage increase rate)

$$
\begin{aligned}
& \gamma=0.002 \frac{\mathrm{mm}^{3}}{\text { cells }} \quad \text { (inverse of maximal macrophage density) } \\
& \eta=0.07 \frac{\mathrm{pg}}{\text { cells } \cdot \text { day }} \quad(\mathrm{TGF}-\beta \text { production rate }) \\
& K(T)=-2.47 T^{3}+21.94 T^{2}+6.41 T+1.75 \frac{\text { cells }}{\mathrm{day} \cdot \mathrm{mm}^{3}} \\
& \text { (rate of incoming macrophages due to } T \text { ). }
\end{aligned}
$$

Observe that $\delta_{2} \simeq D_{2} \gg 1$. After the non-dimensional changes given in Section 2 , the new values for the non-dimensional parameters are

$$
\begin{array}{r}
D=9.1, \quad H=0.18615, \quad \delta_{1}=5.772, \quad \delta_{2}=262.626 \\
K(T)=-1.469325874 \cdot 10^{8} T^{3}+1.292090671 \cdot 10^{6} T^{2} \\
+3.737227980 \cdot 10^{2} T+1.010101010 \cdot 10^{-1} .
\end{array}
$$




\section{References}

[1] A. N. Carvalho, S. M. Oliva, A. L. Pereira, and A. RodríGUEZ-BERnAL, Attractors for parabolic problems with nonlinear boundary conditions, J. Math. Anal. Appl. 207(2) (1997), 409-461. DOI: $10.1006 /$ jmaa. 1997.5282 .

[2] N. El Khatib, S. Génieys, B. Kazmierczak, and V. Volpert, Mathematical modelling of atherosclerosis as an inflammatory disease, Philos. Trans. R. Soc. Lond. Ser. A Math. Phys. Eng. Sci. 367(1908) (2009), 4876-4886. DOI: 10.1098/rsta.2009.0142.

[3] N. El Khatib, S. Genieys, B. Kazmierczak, and V. Volpert, Reaction-diffusion model of atherosclerosis development, J. Math. Biol. 65(2) (2012), 349-374. DOI : 10.1007/s00285-011-0461-1.

[4] D. Henry, "Geometric theory of semilinear parabolic equations", Lecture Notes in Mathematics 840, Springer-Verlag, Berlin-New York, 1981.

[5] H. J. KuIPER, Positively invariant regions for strongly coupled reaction-diffusion systems with a balance law, J. Math. Anal. Appl. 249(2) (2000), 340-350. DOI: 10.1006/jmaa.2000.6835.

[6] O. A. LadyžEnskaja and N. N. URAL'Ceva, "Equations aux dérivées partielles de type elliptique", Traduit par G. Roos, Monographies Universitaires de Mathématiques 31, Dunod, Paris, 1968.

[7] J.-L. Lions and E. Magenes, Problemi ai limiti non omogenei. V, Ann. Scuola Norm Sup. Pisa (3) 16 (1962), 1-44.

[8] S. McDougall, J. Dallon, J. Sherratt, and P. Maini, Fibroblast migration and collagen deposition during dermal wound healing: mathematical modelling and clinical implications, Philos. Trans. R. Soc. Lond. Ser. A Math. Phys. Eng. Sci. 364(1843) (2006), 1385-1405. DOI: 10.1098/rsta.2006.1773.

[9] L. Olsen, P. K. Maini, J. A. Sherratt, and B. Marchant, Simple modelling of extracellular matrix alignment in dermal wound healing. I. Cell flux induced alignment, J. Theor. Med. 1 (1998), 175-192.

[10] M. H. Protter and H. F. Weinberger, On the spectrum of general second order operators, Bull. Amer. Math. Soc. 72 (1966), 251-255. DOI: 10.1090/S0002-9904-1966-11485-4.

[11] J. A. SherratT, A comparison of periodic travelling wave generation by Robin and Dirichlet boundary conditions in oscillatory reaction-diffusion equations, IMA J. Appl. Math. 73(5) (2008), 759-781. DOI: 10.1093/imamat/hxn015. 
[12] J. A. Sherratt and J. D. Murray, Models of epidermal wound healing, Proc. R. Soc. Lond. B. 241 (1990), 29-36. DOI: $10.1098 / \mathrm{rspb} .1990 .0061$.

[13] A. J. Singer and R. A. F. Clark, Mechanisms of disease: cutaneous wound healing, N. Engl. J. Med. 341 (1999), 738-746. DOI: 10.1056/NEJM199909023411006.

[14] R. T. Tranquillo and J. D. Murray, Continuum model of fibroblast-driven wound contraction: Inflammation-mediation, $J$. Theor. Biol. 158(2) (1992), 135-172.

[15] H. V. Waugh and J. A. Sherratt, Macrophage dynamics in diabetic wound healing, Bull. Math. Biol. 68(1) (2006), 197-207. DOI : $10.1007 / \mathrm{s} 11538-005-9022-3$.

[16] H. V. Waugh and J. A. Sherrate, Modeling the effects of treating diabetic wounds with engineered skin substitutes, Wound Repair Regen. 15(4) (2007), 556-565. DOI: 10.1111/j.1524-475X.2007. 00270.x.

N. Cónsul:

Departament de Matemàtica Aplicada I

Universitat Politècnica de Catalunya

ETSEIB

Av. Diagonal 647

Barcelona 08028

Spain

E-mail address: neus.consul@upc.edu

S. M. Oliva:

Departamento de Matemática Aplicada

IME, USP

Rua do Matão, 1.010

CEP 05508-900, São Paulo, SP

Brazil

E-mail address: smo@ime.usp.br

M. Pellicer:

Departament d'Informàtica, Matemàtica Aplicada i Estadística

Universitat de Girona

Campus Montilivi, EPS, Ed. P4

Girona 17071

Spain

E-mail address: martap@imae.udg.edu

Primera versió rebuda el 25 de setembre de 2012, darrera versió rebuda el 22 d'octubre de 2013. 